\title{
Participación y satisfacción en un programa bioecológico en Educación Física en función del sexo, edad y necesidad educativa en un centro de compensatoria
}

\section{Participation and satisfaction in a bioecological program in Physical Education according to sex, age and educational need in a compensatory centre}

\author{
Javier Lamoneda Prieto
}

Grupo de investigación "Physical Activity for Health Promotion", Universidad de Granada. Consejería de Educación. Junta de Andalucía (España).

\begin{abstract}
Resumen: El propósito del presente estudio es analizar los niveles de participación y satisfacción en un programa de Educación Física siguiendo un modelo bioecológico en función del sexo, la edad y la necesidad educativa. Participaron 136 estudiantes con una edad media de 14.25 ańos (DT = 1.66), todos pertenecientes a un centro público ubicado en una zona en riesgo de exclusión. Los resultados revelaron que los niveles de participación no dependían del sexo, edad o necesidad educativa. Los valores de participación atenta y falta de asistencia no fueron positivos (participó un 55.3\% y faltó el $45.6 \%$ ); sin embargo, la participación relativa sí fue satisfactoria (entre un 68 y 90\%), así como el disfrute (89.8\%) y valor del programa para la vida personal (93.2\%). Se sugiere replantear los programas de compensación educativa dando protagonismo a la Educación Física desde una orientación bioecológica.

Palabras Clave: compensación educativa; adolescencia; participación; Educación Física.
\end{abstract}

Abstract: The purpose of this study is to analyze the levels of participation and satisfaction in a Physical Education program following a bioecological model according to sex, age and educational need. A total of 136 students with an average age of 14.25 years $(\mathrm{DT}=1.66)$ participated, all belonging to a public centre located in an area at risk of exclusion. The results revealed that the levels of participation did not depend on sex, age or educational need. The values of attentive participation and lack of attendance were not positive (55.3\% participated and $45.6 \%$ were absent); however, relative participation was satisfactory (between 68 and 90\%), as was enjoyment $(89.8 \%)$ and value of the program for personal life (93.2\%). It is suggested that educational compensation programs be rethought, giving prominence to Physical Education from a bioecological orientation.

Keywords: educational compensation; adolescence; participation; Physical Education.

\section{Introducción}

Uno de los principales retos del sistema educativo actual es considerar precisamente la función que esta cumple en contextos de exclusión social, en los que se ha demostrado un alto índice de absentismo (Junta de Andalucía, 2011).

En lo referente a la Educación Compensatoria, se caracteriza por dirigirse a aquellos sujetos que, a causa de su origen socioeconómico, padecen, o se encuentran en situación de riesgo de padecer, dificultades en su desarrollo educativo. En este contexto, la enseńanza no sólo se aborda como un ejercicio pedagógico con el fin de compensar carencias académicas o de aprendizaje, sino que contempla otros factores influyentes en la misma. Para ello, se requiere de un proceso sistemático y planificado desde su inicio y busca una fuerte relación entre agentes y sujetos; así mismo, de intervenciones interdisciplinares de un enfoque ecológico (Fernández, Huete y Vélez, 2017; Lamoneda, 2020a).

Para el logro de buenos climas de grupo, el fomento de la integración, la convivencia, la participación y la resolución pacífica de conflictos, así como el desarrollo de niveles de responsabilidad en adolescentes y grupos en riesgo de exclusión desde el ámbito de la actividad física y el deporte se han adoptado numerosas medidas que pueden agruparse en cuatro (Capllonch y Figueras, 2012; Lamoneda, 2017; Lamoneda, 2020a; López-Pastor, 2012; Martinek y Hellison, 2009; Ríos, 2009; Soler, Flores, y Prat, 2012): estrategias psico-pedagógicas, programas, modelos de enseńanza-aprendizaje y adaptaciones (figura 1). 


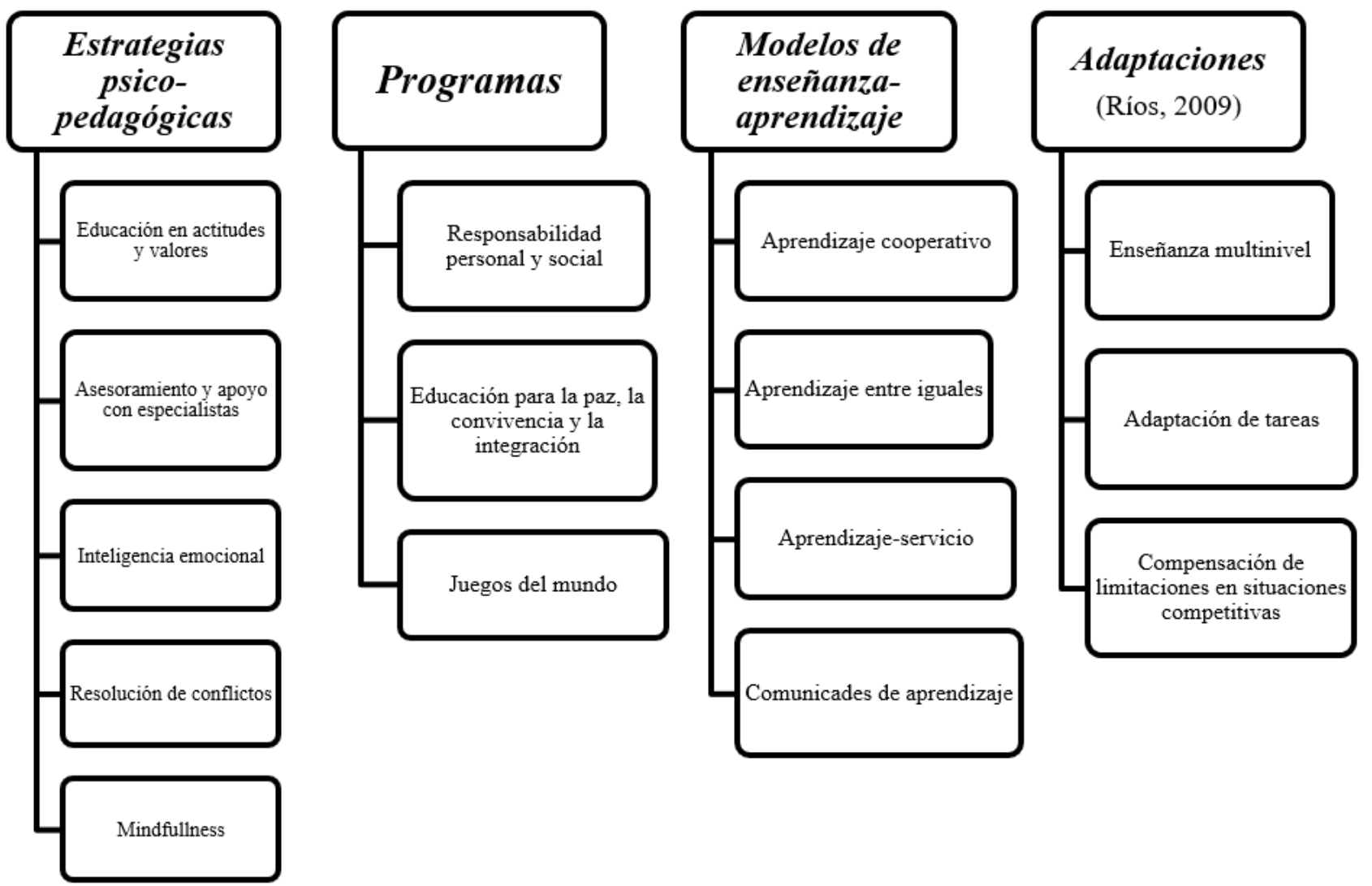

Figura 1. Estrategias de intervención en el deporte e inclusión (elaboración propia).

Entre las estrategias metodológicas empleadas con jóvenes nes (Consejo Superior de Deportes, 2010) (figura 2). en riesgo de exclusión social cabe mencionar seis orientacio-

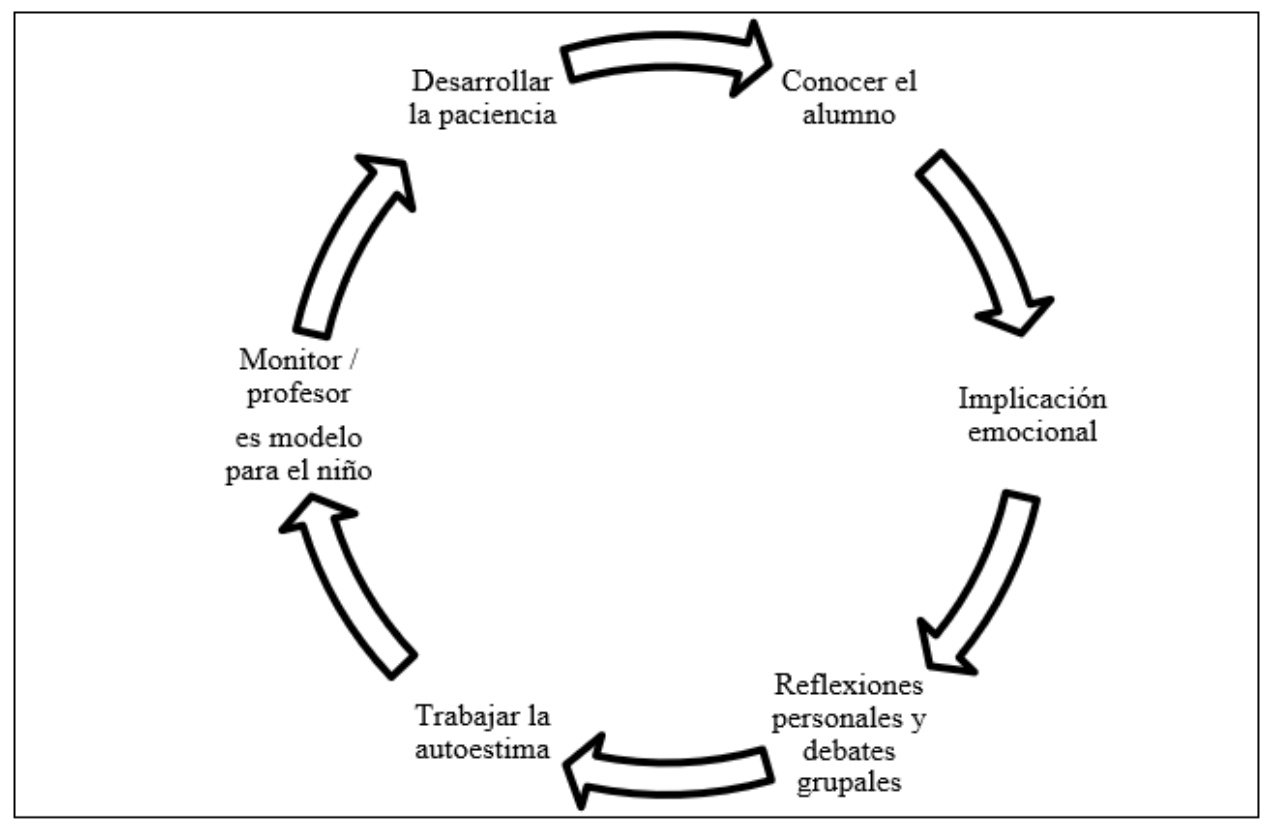

Figura 2. Orientaciones metodológicas en la intervención como educador inclusivo (mod. CSD, 2010). 
1. Conocer a tus alumnos: antes de enseñar conocer de dónde parte cada alumno/a: sus preocupaciones diarias, problemas familiares, gustos, intereses, posibilidades o limitaciones. En la medida de lo posible preocuparse a diario por los intereses de los demás, por encima de los objetivos curriculares.

2. Tener claro hasta dónde te quieres implicar emocionalmente: la confianza puede ayudar a que empieces a recibir información delicada o emocionalmente impactante. Por ello, es importante mantener una distancia emocional como profesional, siendo consciente de que no puedes resolver las situaciones de vida tan debilitadas. En este sentido se requiere de un trabajo multidisciplinar con psicólogos o educadores sociales.

3. Establecer reflexiones personales y debates en grupo: la única forma de cambiar los valores o actitudes de una persona es hablando con ella y ofreciéndole experiencias que la obliguen a desarrollar un razonamiento moral.

4. Potenciar siempre su autoestima: resaltando lo que hacen bien, valorando sus esfuerzos, animándoles y reconociendo lo que hacen constantemente, incluso diciéndoles las cosas que hacen que te alegres.

5. Toma conciencia de que eres un modelo para tus alumnos/as: tener siempre presente que todo lo que expreses en tus actividades afecta a los chicos/as y que siempre están pendientes de tu comportamiento. Si el docente establece unas normas es él el primero que debe cumplirlas y ser buen modelo para ellos.

6. Desarrollar la paciencia: un gran porcentaje de los alumnos/as llevan conviviendo en situaciones de vida durante los 14 o 16 años de edad que tienen, y en unas sesiones o un año de intervención es complicado que cambien radicalmente.

Entre otras teorías explicativas del desarrollo humano, el modelo bioecológico ha adquirido protagonismo en su uso contemporáneo en las ciencias psicológicas y pedagógicas (Gifre y Esteban, 2012). Según el modelo que originalmente aporta Brefenbrenner el desarrollo personal es fruto de un conjunto de procesos de interacción entre la persona y el ambiente. De tal modo que la riqueza de la persona es concebida por la cantidad, calidad y diversidad de interrelaciones y vínculos que se establezca.

A pesar del interés por analizar las medidas para mejorar la asistencia y participación de colectivos vulnerables en centros de compensación educativa (Corchuelo, Azánzazu y Tirado, 2019), sigue siendo necesario profundizar en su estudio debido a que se trata de un campo complejo, que precisa de un estudio multidisciplinar (Lamoneda, 2020a). En el ámbito de la actividad física y el deporte se han analizado los niveles de participación en función del sexo y la edad, concluyendo que los varones logran niveles más altos de participación las clases de educación física (EF) que las chicas y como durante la etapa de Secundaria los niveles de práctica de actividad físi- ca tienden a disminuir (Lamoneda y Huertas-Delgado, 2017). Finalmente, se han analizado a los estudiantes diagnosticados con alguna necesidad educativa específica de apoyo educativo (NEAE), principalmente las pautas para la inclusión en clase de EF (Caus, Santos, Blasco, Vega, Mengual y Yagüez, 2013).

Sin embargo, hasta donde hemos podido analizar, no se ha analizado la relación entre las variables sexo, edad y NEAE en la participación de EF, además se ha detectado la necesidad de aportar programas contextualizados a la realidad particular de estudiantes en riesgo de exclusión social. Por todo ello, el propósito de este estudio fue examinar los niveles de participación y satisfacción con un programa de EF siguiendo un modelo bioecológico en función del sexo, la edad y la NEAE.

\section{Método}

La estrategia metodológica empleada en la presente investigación fue el estudio de caso, que como considera Eisenhardt (1989) permite comprender las dinámicas presentes en contextos singulares.

\section{Participantes}

136 estudiantes con una edad media de 14.25 años, 76 chicos (55.9\%) y 60 chicas (44.1\%); todos pertenecientes a un centro público ubicado en una zona en riesgo de exclusión y que sigue un Programa de Compensación Educativa (Ley 17/2007, de 10 de diciembre, de Educación de Andalucía). Entre los estudiantes, 90 no habían sido diagnosticados con alguna NEAE (66.2\%) y 46, sí (33.8\%).

\section{Procedimiento}

Se dedicaron los diez últimos minutos de cada sesión a consensuar con los estudiantes el nivel de participación durante un total de cincuenta sesiones de EF que siguieron un programa de desarrollo positivo bajo el modelo bioecológico (Lamoneda, 2019).

Para ello, se diseńó un programa original siguiendo el modelo de desarrollo humano de Brefenbrenner (Lamoneda, 2020a). Considerando que el desarrollo es fruto de la interacción del individuo en un contexto o ambiente ecológico, se analizaron los posibles espacios de práctica de actividad física que permitiesen generar interacción y vínculos. En orden a las estructuras seriadas de Brefenbrenner (1987) los ambientes ecológicos en EF fueron un total de cinco: (1) Sesión de EF, (2) Actividades recreativas en tiempo de recreo; (3) Actividades deportivas en el barrio: encuentros intercentro; (4) Contacto con entidades deportivas locales: centros fitness, clubes, delegación municipal, ONGs; (5) Actividades de aventura en el medio natural en entornos lejanos. 


\section{Instrumentos}

Comportamientos de responsabilidad personal

Se registró los comportamientos de responsabilidad personal a través del cuaderno del profesor. Para ello, se tomó como referencia el modelo de Balaguer (2017). Los eventos observados en la hora de registro fueron cinco: falta de asistencia, lesión, apatía, participación discreta y participación atenta (tabla 1).

Tabla 1. Comportamientos de responsabilidad personal (mod. Balaguer, 2017).

\begin{tabular}{|c|c|c|}
\hline Niveles de participación & Indica & Definición \\
\hline \multirow{4}{*}{ No participa } & 1. Falta de asistencia & No asiste a clase \\
\hline & 2. Lesión o enfermedad & No participa por enfermedad justificada \\
\hline & 3. Apatía & No participar, timidez, miedo, cansancuio o desmotivación \\
\hline & 4. Discreta & $\begin{array}{l}\text { Participa de forma itermitente, con distracción, baja intensidad, lentitud e incluso } \\
\text { abandona }\end{array}$ \\
\hline Participa & 5. Atenta & Actuar con intensidad, rapidez, atención durante las actividades y el juego \\
\hline
\end{tabular}

Satisfacción con el programa. Se empleó dos ítems del Cuestionario para el Análisis de la Satisfacción en EF (Moreno, Hellín y Hellín, 2006) sobre el gusto por la actividad realizada e importancia que tenía para su vida.

\section{Análisis estadístico}

En primer lugar se llevaron a cabo estudios descriptivos (medias y desviaciones típicas) y análisis de la normalidad de la muestra. Los resultados de sugirieron abordar el estudio utilizando pruebas no paramétricas.

En segundo lugar, para el análisis de la participación en relación con la edad (10-2o ESO o $3^{\circ}-4^{\circ}$ ESO), sexo (masculino o femenino) y NEAE (sin o con) se analizaron frecuencias y se analizan las diferencias entre grupos, empleando el test exacto de Fisher.

En tercer lugar, se muestra las frecuencias de la evolución de los niveles de participación en el tiempo. En este caso se aporta valores de regresión lineal simple.

Finalmente, sobre la satisfacción en el programa se muestra para el primer ítem, gusto por la actividad realizada, el porcentaje de participantes que asignaron valores de satisfac- ción máxima. En relación con el segundo ítem, importancia que le atribuye, se aportan valores 1 o 2 que corresponden con respuestas "alto" o "muy alto".

Todas las operaciones se realizaron a través del paquete informático para ciencias sociales IBM SPSS 20.0 para Windows co un nivel de significatividad estadística $p \leq .05$.

\section{Resultados}

\section{Niveles de participación en función del curso, sexo y NEAE}

Para los estudiantes la participación en el programa no dependía de la edad, sexo y nivel de NEAE. No se encontraron diferencias significativas en los resultados totales (tabla 3). Sin embargo, se encontraron diferencias en relación con la lesión o la participación apática o discreta. En estos casos los grupos superiores ( $3^{\circ}$ y $4^{\circ}$ ESO) se mostraron más propensos a lesiones y los menores (10 y $2^{\circ}$ ESO) a una participación apática. En relación con el sexo, las chicas se mostraron más propensas que los chicos a la lesión y participar con un rendimiento bajo (apático o discreto). 
Tabla 3. Niveles de participación en relación con el curso, sexo y NEAE.

\begin{tabular}{|c|c|c|c|c|c|c|c|c|c|c|c|}
\hline & \multirow[b]{2}{*}{$\begin{array}{c}M(S D) \\
N=136\end{array}$} & \multirow[b]{2}{*}{ (\%) } & \multicolumn{3}{|c|}{ Curso } & \multicolumn{3}{|c|}{ Sexo } & \multicolumn{3}{|c|}{$N E A E$} \\
\hline & & & $\begin{array}{r}1^{o}-2^{o} \\
N=63\end{array}$ & $\begin{array}{l}3^{o}-4^{o} \\
N=73\end{array}$ & $P$ & $\begin{array}{c}M \\
N=76\end{array}$ & $\begin{array}{c}F \\
N=60\end{array}$ & $p$ & $\begin{array}{c}\text { Sin } \\
N=90\end{array}$ & $\begin{array}{c}C \text { Con } \\
N=46\end{array}$ & $p$ \\
\hline \multicolumn{12}{|c|}{ No participa } \\
\hline Falta & $13.52(11.87)$ & 27.04 & $13.35(10.41)$ & $13.67(13.07)$ & .221 & $12.18(12.33)$ & $15.22(11.14)$ & .987 & $13.22(11.13)$ & $14.11(13.31)$ & .118 \\
\hline Lesión & $.93(1.71)$ & 1.86 & $.49(.91)$ & $1.30(2.11)$ & .001 & $.49(.89)$ & $1.48(2.27)$ & .000 & $.89(1.86)$ & $1(1.38)$ & .989 \\
\hline Apatía & $3.74(5.03)$ & 7.48 & $4.59(6.26)$ & $3.01(3.54)$ & .000 & $1.78(2.85)$ & $6.23(6.02)$ & .000 & 3.34 (4.79) & $4.52(5.43)$ & .346 \\
\hline Discreta & $3.81(3.42)$ & 7.62 & $3.78(3.06)$ & $3.84(3.72)$ & .093 & $2.58(2.53)$ & $5.37(3.76)$ & .000 & $3.62(3.45)$ & $4.17(3.35)$ & .333 \\
\hline Total & $22(13.38)$ & 44.70 & $22.21(14.38)$ & $21.82(12.55)$ & .088 & $17.03(12.43)$ & $28.30(11.87)$ & .555 & $21.08(13.18)$ & $23.80(13.73)$ & .496 \\
\hline \multicolumn{12}{|l|}{ Participa } \\
\hline Total & $27.65(13.66)$ & 55.30 & $27.52(14.63)$ & $27.75(12.87)$ & .098 & $32.66(12.79)$ & $21.30(12.07)$ & .623 & $28.59(13.45)$ & $25.80(14.04)$ & .501 \\
\hline
\end{tabular}

Las figuras 3 y 4 muestran los porcentajes de participación en el programa y falta de asistencia. Con respecto a la participación el $55.3 \%$ lo hizo de forma atenta y el $27.04 \%$ faltó a

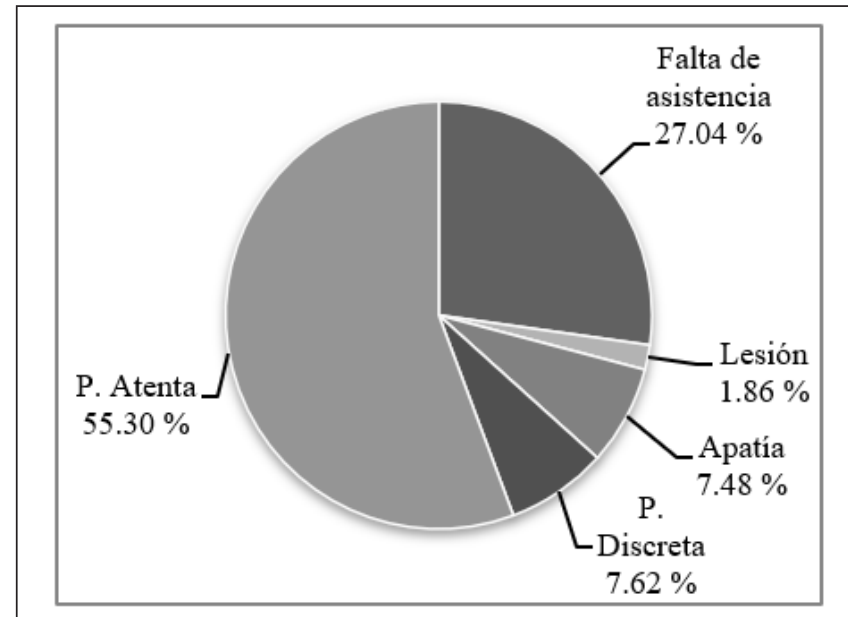

Figura 3. Participación (\%). la sesión. Sobre la falta de asistencia, el $54.4 \%$ faltó menos de un $20 \%$ y el $23.53 \%$ entre un 21 y $40 \%$.

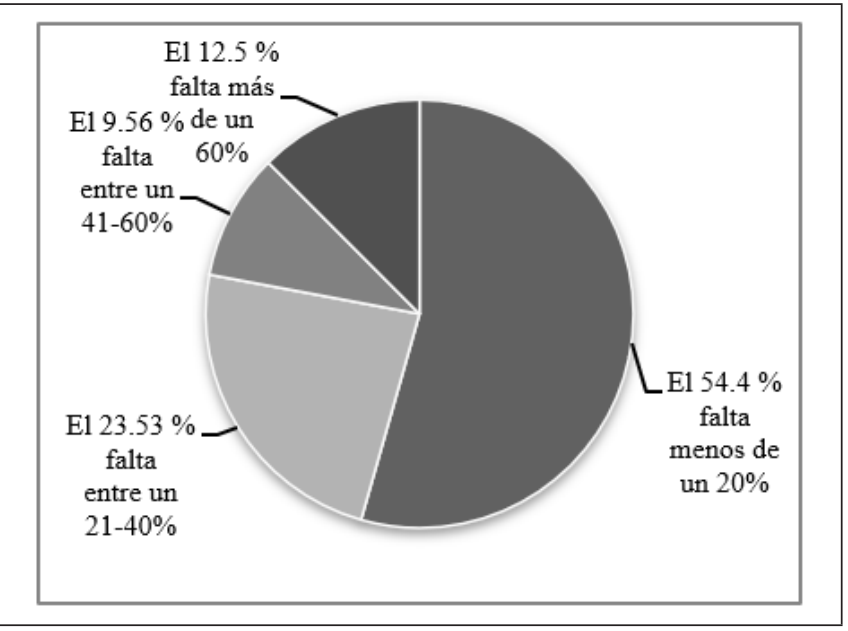

Figura 4. Falta de asistencia (\%).

\section{Evolución de la participación en el programa}

La figura 5 muestra la evolución de la participación atenta relativa (línea continua superior), absoluta (línea discontinua media) y falta de asistencia (línea de puntos inferior).

Sobre la participación atenta absoluta, se aprecia una tendencia decreciente de los valores porcentuales $\left(\mathrm{R}_{2}=0.256\right)$. La participación osciló en valores mínimos de $48.53 \%$ y máximo de $68.38 \%$
En relación a la participación atenta relativa, se realizaron los cálculos porcentuales en relación con los estudiantes que asistían cada semana. La línea de tendencia muestra una estabilidad en la participación $\left(\mathrm{R}_{2}=0.001\right)$. El porcentaje de participación se distribuyó en un rango entre $68.32 \%$ y $90.53 \%$.

Con respecto a la evolución de asistencia, la línea de tendencia temporal muestra una evolución positiva $\left(R_{2}=0.321\right)$. En este caso el rango varió entre un $8.82 \%$ y $34.56 \%$. 


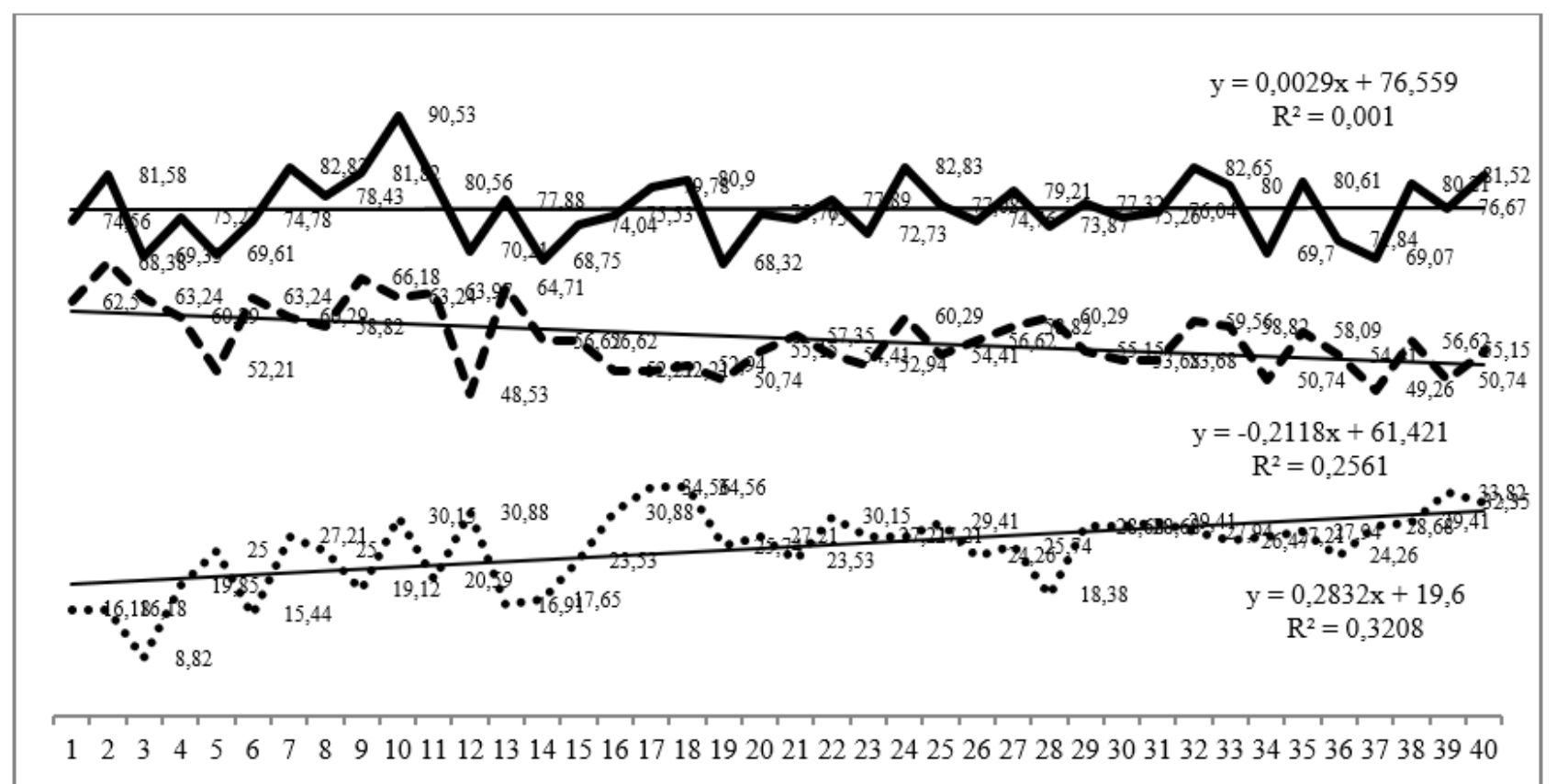

- $\begin{aligned} & \text { Participación } \\ & \text { atenta (relativa) }\end{aligned}-\begin{aligned} & \text { Participación } \\ & \text { absoluta }\end{aligned} \quad \ldots \ldots \ldots \ldots$ Falta de asistencia

Figura 5. Evolución de la participación atenta y falta de asistencia durante el Programa Bioecológico en EF

Valoración del programa

En la tabla 5 podemos comprobar que la satisfacción por el programa bioecológico en EF no es una variable significativa diferencialmente con respecto al curso, sexo o NEAE. Tanto el gusto por la sesión como la importancia que le atribuían al programa fue alta o muy alta: al $89 \%$ le gustó mucho y el 93\% consideró que era importante o muy importante para él (tabla 5).

Tabla 5. Valores porcentuales de la satisfacción de los participantes en el programa Bioecológico en EF

\begin{tabular}{lcccccccccc}
\hline & Total & \multicolumn{3}{c}{ Curso } & \multicolumn{3}{c}{ Sexo } & \multicolumn{3}{c}{ NEAE } \\
\cline { 2 - 10 } & $\boldsymbol{( \% )}$ & $\mathbf{1}^{\boldsymbol{o}-\mathbf{2}^{\boldsymbol{o}}}$ & $\mathbf{3}^{\boldsymbol{o}-\mathbf{4}^{\boldsymbol{o}}}$ & $\boldsymbol{P}$ & $\boldsymbol{M}$ & $\boldsymbol{F}$ & $\boldsymbol{p}$ & Sin & Con & $\boldsymbol{p}$ \\
\hline Le había gustado mucho & 89.8 & 95.12 & 85.11 & .350 & 96.23 & 80 & .073 & 89.83 & 89.66 & .860 \\
Era importante o muy importante & 93.2 & 90.48 & 73.91 & .334 & 92.31 & 73.91 & .084 & 94.92 & 89.66 & .521 \\
\hline
\end{tabular}

\section{Discusión}

El presente estudio ha tenido como objeto examinar los niveles de participación y satisfacción en un programa de EF siguiendo un modelo bioecológico en función del sexo, la edad y la NEAE. Los resultados obtenidos revelaron: (1) que los niveles de participación no dependían de variables como sexo, edad o NEAE; (2) que la participación atenta media no fue satisfactoria (55.3\%), ni la falta de asistencia (el 45.6\% superó el 20\% de ausencia); (3) se apreció un nivel alto de participación atenta en valores relativos (si no se contaba la falta de asistencia), que osciló entre un 68 y 90\%; (4) la mayoría de los participantes revelaron que les había gustado mucho
$(89.8 \%)$ y era importante o muy importante para sus vidas personales (93.2\%).

En la interpretación de trabajos implementados con jóvenes en riesgo de exclusión social conviene ser cautos debido a las grandes carencias que cuenta este colectivo con consumo de drogas, conducta antisocial, trastornos depresivos o prácticas sexuales de riesgo (Arnett, 1999). Se ha constatado que los jóvenes procedentes de familias de un nivel socioeconómico bajo se ven altamente condicionados en el plano laboral y cultural respecto a los de una condición media (Van Ewijk y Sleegers, 2010). Por todo ello, los valores estadísticos obtenidos son relativos. Como se ha argumentado en otros trabajos el éxito del programa puede venir por la mejora de las 
condiciones de vida de un único chico/a (Lamoneda, 2020). En este sentido, el porcentaje de satisfacción con el programa y la importancia que se le atribuye concede gran valor a la intervención realizada.

Respecto a la ausencia de relación entre las variables estudiadas (sexo, edad, NEAE) con la participación en EF los resultados difieren de los datos obtenidos en otras poblaciones, ya que para un nivel socioeconómico-medio, la edad tenía una relación inversa con la práctica deportiva y el nivel de actividad física en chicos era mayor que en chicas (Lamoneda y Huertas-Delgado, 2017). Los datos obtenidos sugieren que no se precisa de una intervención diferenciada en relación con las tres variables analizadas. La causa de esta ausencia de relación entre variables podemos encontrarla en que en el contexto particular de estudiantes en situación de riesgo de exclusión la participación va a depender de generar un buen clima de trabajo: "que tenga buen trato con los estudiantes, adquiera un alto nivel de compromiso, sea participativo y genere en el grupo relaciones sociales favorables" (Lamoneda, 2020b, p.62), independientemente del sexo, edad o NEAE.

En relación con la falta de asistencia los valores obtenidos no fueron satisfactorios. Estos resultados se relacionan con estudios que muestran el abandono prematuro en jóvenes como uno de los problemas más graves del sistema educativo español (Tarabini, Jacovkis y Montes, 2017). Según los datos de la Encuesta de Población Activa (EPA, 2017), un 18.2\% de los jóvenes entre 18 y 24 años abandonan el sistema educativo; por comunidades autónomas, Andalucía lidera el fracaso con un índice del $23.5 \%$, muy por encima de la media europea, que sitúa la tasa el 10.7\%. Entre otros factores, la condición social es considerada el atributo con más peso; de hecho, se ha marcado un $50 \%$ de diferencias en rendimiento en familias con una mejor posición económica respecto a las de menos recursos (Serrano, Soler, Hernández y Sabater 2013).

El incremento temporal de la falta de asistencia supuso un descenso de los niveles de participación totales que oscilaron entre el $48.53 \%$ y $68.38 \%$. Sin embargo, si en el análisis estadístico nos centramos en los estudiantes que asistían a clase de EF los resultados son esperanzadores, ya que los niveles se incrementaron ligeramente durante el programa $\left(\mathrm{R}_{2}=0.001\right)$ y fueron satisfactorios: entre el $68.32 \%$ y $90.53 \%$ de los alumnos/as que asistían a clase lo hacían de forma atenta. Esos resultados nuevamente ponen de manifiesto la necesidad de relativizar los datos estadísticos generales en este tipo de centros. Sin embargo, siguen mostrando la necesidad de reducir la falta de asistencia al centro ya que en el caso particular de la materia de EF los estudiantes que asisten se muestran interesados.

El presente estudio presenta las limitaciones propias de un estudio de caso, que muestra la realidad particular de un centro, un tipo particular de alumnado que dificultan la generalización de los resultados en tipo de otro contexto al descrito, pero al mismo tiempo, puede ser de interés en el ámbito educativo para abordar la compleja intervención docente en centros de compensación educativa.

\section{Conclusiones}

En primer lugar, este estudio revela que la participación en clase de EF en centros de compensación educativa no depende de variables como el sexo, edad o necesidad educativa, cuando en la población en general sí han sido variables a considerar.

En segundo lugar, se aporta un programa de intervención original basado en el modelo bioecológico de Brefembrenner que se caracteriza por generar ambientes ecológicos de aprendizaje que van desde la clase de educación física, la actividad en tiempo de recreo, prácticas conjuntas con estudiantes del barrio próximo, contacto con entidades locales y acercamiento a espacios naturales. Resulta interesante mostrar que para la mayoría de los participantes el programa les había gustado mucho y había sido importante o muy importante para sus vidas.

En tercer lugar, se pone de manifiesto la preocupante situación que atraviesan jóvenes en riesgo de exclusión social sobre el alto índice de absentismo escolar; si bien, se aporta un hallazgo esperanzador, ya que los estudiantes que asistían a clase de EF lo hacían con interés.

\section{Aplicaciones prácticas}

Este trabajo muestra la necesidad de repensar la educación compensatoria, dando mayor protagonismo a la materia de EF debido al alto interés que presentan los estudiantes independientemente del sexo, edad o NEAE; y aporta una intervención educativa basada en un modelo aún poco explorado en el ámbito de la actividad física y el deporte como el bioecológico.

\section{Referencias}

1. Arnett, J. (1999). Adolescent Storm and Stress, Reconsidered. American Psychologist, 54, 317-326.

2. Balaguer, J. (2017). Estudio de un factor de competencia básica a través del juego Z. Apunts, 128(2), 48-63.

3. Bronfenbrenner, U. (1987). La ecología del desarrollo humano. Experimentos en entornos naturales y diseñados. Barcelona: Paidós.
4. Caus, N., Santos, E., Blasco, J., Vega, L., Mengual, S., y Yangüez, E. (2013). Procedimiento de actuación ante la Inclusión de alumnado con discapacidad en el área de educación física. Apunts, 112(2), 37-45.

5. Capllonch, M., y Figueras, S. (2012). Educación Física y Comunidades de Aprendizaje. Estudios pedagógicos Valdivia, 38, 231-247. doi:10.4067/ S0718-07052012000400013 
6. Consejo Superior de Deportes (2010). Deporte actividad física e inclusión social. Una guía para la intervención social a través de las actividades deportivas. Plan A+D. Programa: Actividad física, deporte e inclusión social. CSD: Madrid.

7. Corchuelo, C., Cejudo, C., y Tirado, R. (2019). Influencia del apoyo familiar y escolar en el compromiso conductual de los estudiantes. Análisis multinivel en un IES de compensación educativa. Revista Complutense De Educación, 30(2), 605-622. doi:10.5209/RCED.57883

8. Eisenhardt, K. M. (1989). Building Theories from Case Study Research. Academy of Management Review, 14(4), 532-550.

9. Fernández, J., Huete, A. y Vélez, L. (2017). Vulnerable groups at risk for sport and social exclusion. Journal of Physical Education and Sport, 17(1), 312-326. doi:10.7752/jpes.2017.01047

10. Gifre, M. y Esteban, M. (2012). Consideraciones educativas de la perspectiva ecológica de Urie Bronfenbrenner. Contextos Educativos, 15, 7992.

11. Junta de Andalucía (2007). Ley 17/2007, de 10 de diciembre, de Educación de Andalucía (BOJA no 252, de 26 de diciembre).

12. Lamoneda, J. (2017). Intervención para educar a adolescentes en una cultura de paz a través del deporte en contextos de exclusión. E-balonmano. Revista de Ciencias del Deporte, 13(3), 225-236.

13. Lamoneda, J. (2019). Responsabilidad, violencia percibida y prosocialidad en adolescentes que precisan atención compensatoria. SPORT TK: Revista Euroamericana de Ciencias del Deporte, 8(2), 27-34

14. Lamoneda, J. (2020a). Aplicación del modelo bioecológico en educación física en contextos de exclusión social. Didacticae, 7. doi:10.1344/ did. 2020.7

15. Lamoneda, J. (2020b). Repercusiones de un programa bioecológico en la responsabilidad y satisfacción en educación física: un estudio de caso en contextos de exclusión social. Espiral. Cuadernos del Profesorado, 13(26), 54-63.

16. Lamoneda, J. y Huertas-Delgado, F. J. (2017). Análisis de la práctica deportiva-recreativa a través de un programa de promoción en el recreo en función del sexo en adolescentes espańoles. Retos: nuevas tendencias en educación física, deporte y recreación, 32, 25-29.

17. López, V.M. (2012). Didáctica de la educación física, desigualdad y transformación social. Estudios Pedagógicos XXXVIII, 1, 155-176.

18. Martinek, T., y Hellison, D. (2009). Youth leadership in sport and physical education. Palgrave MacMillan: Nueva York.

19. Moreno, J. A., Hellín, P., y Hellín, G. (2006). Pensamiento del alumno sobre la educación física según la edad. Apunts: Educación Física y Deportes, $85,28-35$

20. Ríos, M. (2009). La Inclusión en el Área de Educación Física en España. Análisis de las Barreras para la Participación y Aprendizaje. Ágora para la EF y el Deporte, 9, 83-114.

21. Serrano, L., Soler, A., Hernández, L., y Sabater, S. (2013). El abandono educativo temprano: análisis del caso español. Madrid: INEE 290.

22. Soler, S., Flores, G. y Prat, M. (2012). La educación física y el deporte como herramientas de inclusión de la población inmigrante en cataluña: el papel de la escuela y la administración local. Pensar a Prática, Goiânia, 15(1), 1-19. doi:10.5216/rpp.v15i1.16653

23. Tarabini, A., Jacovkis, J., y Montes, A. (2017). Los factores de la exclusión educativa en España: Mecanismos, perfiles y espacios de intervención. Madrid: Unicef Comité Español.

24. Van Ewijk, R. y Sleegers, P. (2010). The effect of peer socioeconomic status on student achievement: a meta-analysis. Educational Research Review, 5(2), 134-150. 